\title{
Upregulation of IncRNA GATA6-AS suppresses the migration and invasion of cervical squamous cell carcinoma by downregulating MTK-1
}

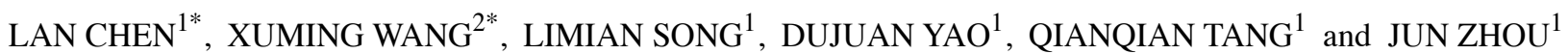 \\ ${ }^{1}$ Department of Obstetrics and Gynecology, Union Hospital, Tongji Medical College, \\ Huazhong University of Science and Technology, Wuhan, Hubei 430022; ${ }^{2}$ Department of Pathology and \\ Pathophysiology, School of Medicine, Jianghan University, Wuhan, Hubei 430056, P.R. China
}

Received June 21, 2018; Accepted April 29, 2019

DOI: $10.3892 / \mathrm{ol} .2019 .10554$

\begin{abstract}
The diagnosis and treatment of cervical squamous cell carcinoma is challenged by difficulties in the determination of tumor invasion. GATA binding protein 6 antisense (GATA6-AS) is a recently identified long non-coding RNA that exhibits critical functions in the growth of endothelial cells; however, to the best of our knowledge, its involvement in other physiological and pathological processes is unknown. By reverse transcription-quantitative polymerase chain reaction, the present study examined the expression of GATA6-AS in tumor tissues and adjacent healthy tissues, and the serum from patients with cervical squamous cell carcinoma and healthy controls. The diagnostic and prognostic potentials of GATA6-AS for cervical squamous cell carcinoma were analyzed by receiver operating characteristic curve analysis and survival curve analysis, respectively. Associations between the serum levels of GATA6-AS and clinical characteristics of patients with cervical squamous cell carcinoma were analyzed by a $\chi^{2}$ test. A GATA6-AS overexpression vector was transfected into cervical squamous cell carcinoma cells, and the effects on cell migration and invasion were investigated by Transwell migration and invasion assays, respectively. The expression of mitogen-activated protein kinase kinase 4 (MTK-1) following transfection with the GATA6-AS overexpression vector was detected by western blot analysis. It was identified that the expression levels of GATA6-AS were lower
\end{abstract}

Correspondence to: Dr Jun Zhou, Department of Obstetrics and Gynecology, Union Hospital, Tongji Medical College, Huazhong University of Science and Technology, 1277 Jiefang Avenue, Wuhan, Hubei 430022, P.R. China

E-mail: rxxqwr9@163.com

*Contributed equally

Key words: cervical squamous cell carcinoma, long non-coding RNA GATA binding protein 6 antisense, mitogen-activated protein kinase kinase 4 , migration, invasion in tumor tissues compared with healthy tissues. In addition, serum levels of GATA6-AS were higher in patients compared with healthy controls. The serum levels of GATA6-AS were associated with tumor metastasis, and may serve as a potential diagnostic and prognostic marker for cervical squamous cell carcinoma. Furthermore, GATA6-AS overexpression inhibited cancer cell migration and invasion. The expression levels of MTK-1 were also reduced following GATA6-AS overexpression. Therefore, the present study proposed that downregulated GATA6-AS expression was associated with tumor metastasis in cervical squamous cell carcinoma, and that GATA6-AS expression may inhibit cancer cell migration and invasion by downregulating MTK-1.

\section{Introduction}

Cervical cancer is one of the most common types of cancer and is the leading cause of cancer-associated mortality in females worldwide (1). Cervical squamous cell carcinoma is the most common type of cervical cancer and accounts for $\sim 80 \%$ of all cases (2). It has been demonstrated that $\sim 90 \%$ of cervical squamous cell carcinoma cases are associated with human papillomavirus (HPV) infection (3). The incidence rate of cervical squamous cell carcinoma has decreased significantly during the last century following the popularization of HPV infection screening programs and increased rates of HPV vaccination (4). However, the occurrence of cervical squamous cell carcinoma is also associated with other factors, and the incidence rate of this disease has demonstrated no significant decrease in last several decades (5). Compared with HPV-positive cervical cancer, the prognosis of HPV-negative cervical cancer is worse (6).

Activation of mitogen-activated protein kinase signaling serves pivotal roles in the development of different types of malignancy $(7,8)$. As a key member of the mitogen-activated protein kinase signaling pathway, mitogen-activated protein kinase kinase 4 (MTK-1) participates in critical cell developmental processes and functions (9). It has been demonstrated that long non-coding RNAs (lncRNAs), a subgroup of non-coding RNAs composed of $>200$ nucleotides, are critical regulators of mitogen-activated protein kinase in 
cancer development (10). GATA binding protein 6 antisense (GATA6-AS) is a recently identified lncRNA that is involved in the regulation of the growth of endothelial cells (11); however, to the best of our knowledge, the involvement of GATA6-AS in other physiological or pathological processes remains unknown. Our preliminary microarray data revealed that GATA6-AS is downregulated in tumor tissues of patients with cervical squamous cell carcinoma (data not shown), which indicates a possible involvement of GATA6-AS in this disease. The present study further confirmed that GATA6-AS is downregulated in cervical squamous cell carcinoma. In addition, GATA6-AS overexpression was demonstrated to inhibit the migration and invasion of cervical squamous cell carcinoma cells and downregulate the expression of MTK-1. To the best of our knowledge, the current study is the first to report the involvement of GATA6-AS in cervical squamous cell carcinoma. In summary, the present study provides novel insights into the pathogenesis of cervical squamous cell carcinoma.

\section{Materials and methods}

Subjects. Between January 2012 and January 2013, a total of 68 female patients with cervical squamous cell carcinoma were diagnosed by pathological examinations at Union Hospital, Tongji Medical College, Huazhong University of Science and Technology (Wuhan, China). The exclusion criteria were as follows: i) Patients with other types of malignancy; ii) patients with other severe diseases; and iii) patients treated prior to admission. The inclusion criteria were as follows: i) Patients pathologically diagnosed with cervical squamous cell carcinoma; ii) patients diagnosed and treated for the first time; and iii) patients willing to participate in the study. According to patient medical records, 18 were HPV-negative and 50 were HPV-positive (20 cases of HPV-11, 14 cases of HPV-16 and 16 cases of HPV-18). The age range of the patients was 24-66 years, with a mean age of $45.3 \pm 4.6$ years. In addition, 42 healthy females (25-67 years; mean age, $44.1 \pm 4.9$ years) were enrolled during the same time period to serve as healthy controls. Age, body mass index and other basic information were compared between patients and controls, and no significant differences were observed. The present study was approved by the Ethics Committee of Union Hospital, Tongji Medical College, Huazhong University of Science and Technology. Written informed consent was obtained from all participants.

Specimen collection. Tumor tissue samples (from three different sites) and adjacent healthy tissue samples (from three different sites) within $2 \mathrm{~cm}$ of the tumor were collected from all patients by biopsy. In addition, serum samples were prepared by centrifuging blood collected from the left arm of all patients and healthy controls at $1,200 \mathrm{x}$ g for $15 \mathrm{~min}$ at room temperature. All samples were stored in liquid nitrogen prior to use.

Cell lines, cell culture and transfection. The occurrence of cervical squamous cell carcinoma is significantly associated with HPV infection (4). Therefore, the present study employed the two human cervical squamous cell carcinoma cell lines SiHa [HPV-positive; cat. no. HTB-35 ${ }^{\mathrm{TM}}$, American Type Culture Collection (ATCC), Manassas, VA, USA] and C33A (HPV-negative; cat. no. HTB-31 ${ }^{\mathrm{TM}}$; ATCC), and the two normal cervical cell line; Ect1/E6E7 (HPV-positive; cat. no. CRL 2614 ${ }^{\mathrm{TM}}$; ATCC) and HCvEpC (HPV-negative; cat. no. 233-75a; Cell Applications, San Diego, CA, USA). Eagle's Minimum Essential Medium supplemented with 10\% FBS (Sangon, Shanghai, China) was used to cultivate the cells at $37^{\circ} \mathrm{C}$ with $95 \%$ humidity and $5 \% \mathrm{CO}_{2}$.

Full GATA6-AS complementary DNA (cDNA) fragments surrounded by EcoRI restriction sites were obtained by PCR amplification and inserted into a pIRSE2-EGFP vector (Clontech Laboratories, Inc., Mountainview, CA, USA) to construct the GATA6-AS overexpression vector. Primers used in PCR amplification were: GATA6-AS forward, 5'-GAATTC AACCCCCGCCTAGCCCTT-3' and reverse, 5'-GAATTC CTGTCTGAATCAACTGTACGA-3'. Phusion ${ }^{\circledR}$ High-Fidelity DNA Polymerase kit (New England BioLabs, Inc., Ipswich, MA, USA) was used to perform PCR amplification under the following thermocycling conditions: $95^{\circ} \mathrm{C}$ for $2 \mathrm{~min}$, followed by 30 cycles of $15 \mathrm{sec}$ at $95^{\circ} \mathrm{C}, 15 \mathrm{sec}$ at $55^{\circ} \mathrm{C}$ and $80 \mathrm{sec}$ at $72^{\circ} \mathrm{C}$. The GATA6-AS expression vector or an empty pIRSE2-EGFP vector (negative control) were transfected into $\mathrm{SiHa}, \mathrm{C} 33 \mathrm{~A}$, Ect1/E6E7 and HCvEpC cells at a concentration of $10 \mathrm{nM}$ using Lipofectamine ${ }^{\circledR} 2000$ Transfection Reagent (Thermo Fisher Scientific, Inc., Waltham, MA, USA). Untransfected cells were used as control cells. Overexpression of GATA6-AS was confirmed by reverse transcription-quantitative PCR (RT-qPCR) prior to subsequent experiments. Following experiments were performed at $24 \mathrm{~h}$ post-transfections.

Transwell migration and invasion assays. Following transfection, $\mathrm{SiHa}, \mathrm{C} 33 \mathrm{~A}, \mathrm{Ect1} / \mathrm{E} 6 \mathrm{E} 7$ and HCvEpC cells were used to generate cell suspensions $\left(5 \times 10^{4}\right.$ cells $\left./ \mathrm{ml}\right)$ in serum-free RPMI-1640 medium (Thermo Fisher Scientific, Inc.). Subsequently, $0.1 \mathrm{ml}$ cell suspension containing $5 \times 10^{3}$ cells was added to the upper chamber of Transwell plates, and RPMI-1640 medium (Thermo Fisher Scientific, Inc., Waltham, MA, USA) supplemented with $20 \%$ fetal calf serum (Sigma-Aldrich; Merck KGaA, Darmstadt, Germany) was added to the lower chamber. Cell migration was analyzed after $24 \mathrm{~h}$. Membranes were then collected and stained with $0.5 \%$ crystal violet (Sigma-Aldrich; Merck KGaA) for $40 \mathrm{~min}$ at $22^{\circ} \mathrm{C}$. For the invasion assay, Matrigel (cat. no. 356234; EMD Millipore, Billerica, MA, USA) was used to coat the upper chamber prior to the addition of cells. All other steps were the same. Cells were counted under a light microscope and 5 visual fields were randomly selected under $\mathrm{x} 40$ magnification. Cell migration and invasion were normalized to the control group, which was set to 100 .

$R T$ - $q P C R$. TRIzol ${ }^{\circledR}$ reagent (Invitrogen; Thermo Fisher Scientific, Inc.) was used to extract total RNA from serum and $\mathrm{SiHa}, \mathrm{C} 33 \mathrm{AE}$, ct1/E6E7 and $\mathrm{HCvEpC}$ cells. For complete cell lysis, tissues were ground in liquid nitrogen prior to adding TRIzol reagent. cDNA was synthesized by RT using AMV Reverse Transcriptase kit (Promega Corporation, USA) and the following conditions: $25^{\circ} \mathrm{C}$ for $5 \mathrm{~min}, 55^{\circ} \mathrm{C}$ for $30 \mathrm{~min}$ and $75^{\circ} \mathrm{C}$ for $10 \mathrm{~min}$. $\mathrm{SYBR}^{\circledR}$ Green Real-Time PCR Master mix (Thermo Fisher Scientific, Inc.) was used 
for the PCR reaction. The following primers were used for PCR: GATA6-AS forward, 5'-ATGCGCTTTTTGCCCTGA AG-3' and reverse, 5'-AGGTCAGCTGGGGAATGTTG-3'; and $\beta$-actin forward, 5'-GACCTCTATGCCAACACAGT-3' and reverse, 5'-AGTACTTGCGCTCAGGAGGA-3'. The PCR conditions were as follows: $95^{\circ} \mathrm{C}$ for $50 \mathrm{sec}$, followed by 40 cycles of $95^{\circ} \mathrm{C}$ for $12 \mathrm{sec}$ and $55^{\circ} \mathrm{C}$ for $35 \mathrm{sec}$. Reactions were performed using the CFX384 Touch $^{\mathrm{TM}}$ Real-Time PCR Detection System (Bio-Rad Laboratories, Inc., Hercules, CA, USA). GATA6-AS expression was normalized to $\beta$-actin using the $2^{-\Delta \Delta \mathrm{Cq}}$ method (12).

Western blotting. Total protein extraction from $\mathrm{SiHa}, \mathrm{C} 33 \mathrm{AE}$, ct1/E6E7 and HCvEpC cells was performed using radioimmunoprecipitation assay solution (Thermo Fisher Scientific, Inc.). Protein concentration was measured by BCA assay. Proteins were separated by $10 \%$ SDS-PAGE with $30 \mu \mathrm{g}$ protein per lane. Gel transfer to polyvinylidene difluoride membranes was performed and blocking was achieved with 5\% skimmed milk at room temperature for $90 \mathrm{~min}$. Subsequently, the membranes were incubated with anti-MTK-1 antibody $(1: 1,200$; cat. no. ab186125; Abcam, Cambridge, UK) or anti-GAPDH endogenous control antibody (1:1,200; cat. no. ab37168; Abcam) at $4^{\circ} \mathrm{C}$ overnight. Membranes were then further incubated with anti-rabbit IgG-horseradish peroxidase secondary antibody (1:1,000; cat. no. MBS435036; MyBioSource, San Diego, CA, USA) for $2 \mathrm{~h}$ at room temperature. Signals were developed using enhanced chemiluminescence (Sigma-Aldrich; Merck $\mathrm{KGaA}$ ) and MTK-1 expression was normalized to GAPDH using ImageJ v.146 software (National Institute of Health, Bethesda, MD, USA).

Statistical analysis. All statistical analyses were performed using SPSS 19.0 (IBM Corp., Armonk, NY, USA) statistical software. All experiments were performed in triplicate and mean values (mean \pm SD) were calculated. Associations between serum GATA6-AS levels and clinicopathological data of patients were analyzed by a $\chi^{2}$ test. Comparisons of protein and RNA expression levels between two groups and among multiple groups were performed by unpaired Student's t-test and one-way analysis of variance followed by Fisher's Least Significance Difference test, respectively. Diagnostic analysis was performed by receiver operating characteristic (ROC) curve analysis. Comparisons of the GATA6-AS expression in tumor tissues and adjacent normal tissues within individuals were performed by a paired Student's t-test. Patients with cervical squamous cell carcinoma were divided into low $(n=34)$ and high $(n=34)$ expression groups according to the median serum level of GATA6-AS. Survival curves were plotted using Kaplan-Meier analysis and compared with a log-rank test. $\mathrm{P}<0.05$ was considered to indicate a statistically significant difference.

\section{Results}

Expression of GATA6-AS in tumor tissues and adjacent normal tissues obtained from HPV-positive and-negative patients. RT-qPCR was performed to measure the expression level of GATA6-AS in tumor tissues and adjacent normal tissues obtained from HPV-positive and -negative patients. In
HPV-negative patients, a significantly lower expression level of GATA6-AS in tumor tissues compared with adjacent normal tissues was observed in 14 out of 18 patients $(\mathrm{P}<0.05$; Fig. 1A). In HPV-positive patients, a significantly lower expression level of GATA6-AS in tumor tissues compared with adjacent normal tissues was observed in 36 out of 50 patients $(\mathrm{P}<0.05$; Fig. 1B).

Serum level of GATA6-AS in patients and healthy controls, and its diagnostic and prognostic value. Serum levels of GATA6-AS in patients and healthy controls were also measured by RT-qPCR. As presented in Fig. 2A, serum levels of GATA6-AS were significantly higher in patients compared with healthy controls $(\mathrm{P}<0.05)$. However, no significant differences were revealed between HPV-negative and HPV-positive patients $(\mathrm{P}>0.05)$. ROC curve analysis was performed to evaluate the diagnostic value of serum GATA6-AS for cervical squamous cell carcinoma. As presented in Fig. 2B, the area under the curve was 0.9373 , with a standard error of 0.02275 and $95 \%$ confidence interval of $0.8927-0.9819$. Patients with cervical squamous cell carcinoma were divided into low $(n=34)$ and high $(n=34)$ expression groups according to the median serum level of GATA6-AS. Survival curves were plotted using Kaplan-Meier analysis and compared with a log-rank test. As presented in Fig. 2C, the survival rate of patients with a high serum GATA6-AS level was significantly increased compared with patients possessing low serum GATA6-AS levels $(\mathrm{P}=0.0006)$.

Associations between serum levels of GATA6-AS and the clinicopathological data of patients. Patients were divided into high $(n=34)$ and low $(n=34)$ GATA6-AS expression groups according to the median serum levels of GATA6-AS. Associations between serum levels of GATA6-AS and clinicopathological data of patients with cervical squamous cell carcinoma were analyzed by a $\chi^{2}$ test. As presented in Table I, no significant associations were identified between serum GATA6-AS levels and age, drinking and smoking habits, or tumor size. However, a significant association was identified between serum GATA6-AS level and tumor distant metastasis.

Effects of GATA6-AS-overexpression on the migration and invasion of human cervical squamous cell carcinoma cells and normal cervical cells. The data presented in Table I suggests GATA6-AS may be involved in distant tumor metastasis of cervical squamous cell carcinoma. Therefore, Transwell cell migration and invasion assays were performed to investigate the effect of GATA6-AS on the migration and invasion of cervical squamous cell carcinoma cells. As presented in Fig. 3, compared with the control and negative control groups, the expression levels of GATA6-AS were significantly higher in all four cell lines following transfection compared with the controls $(\mathrm{P}<0.05$; Fig. 3A). GATA6-AS overexpression significantly inhibited the migration and invasion of the human cervical squamous cell carcinoma cell lines SiHa (Fig. 3B) and C33A (Fig. 3C). However, no significant differences in migration and invasion were identified for the normal cervical cell lines Ect1/E6E7 (Fig. 3D) and HCvEpC (Fig. 3E). 

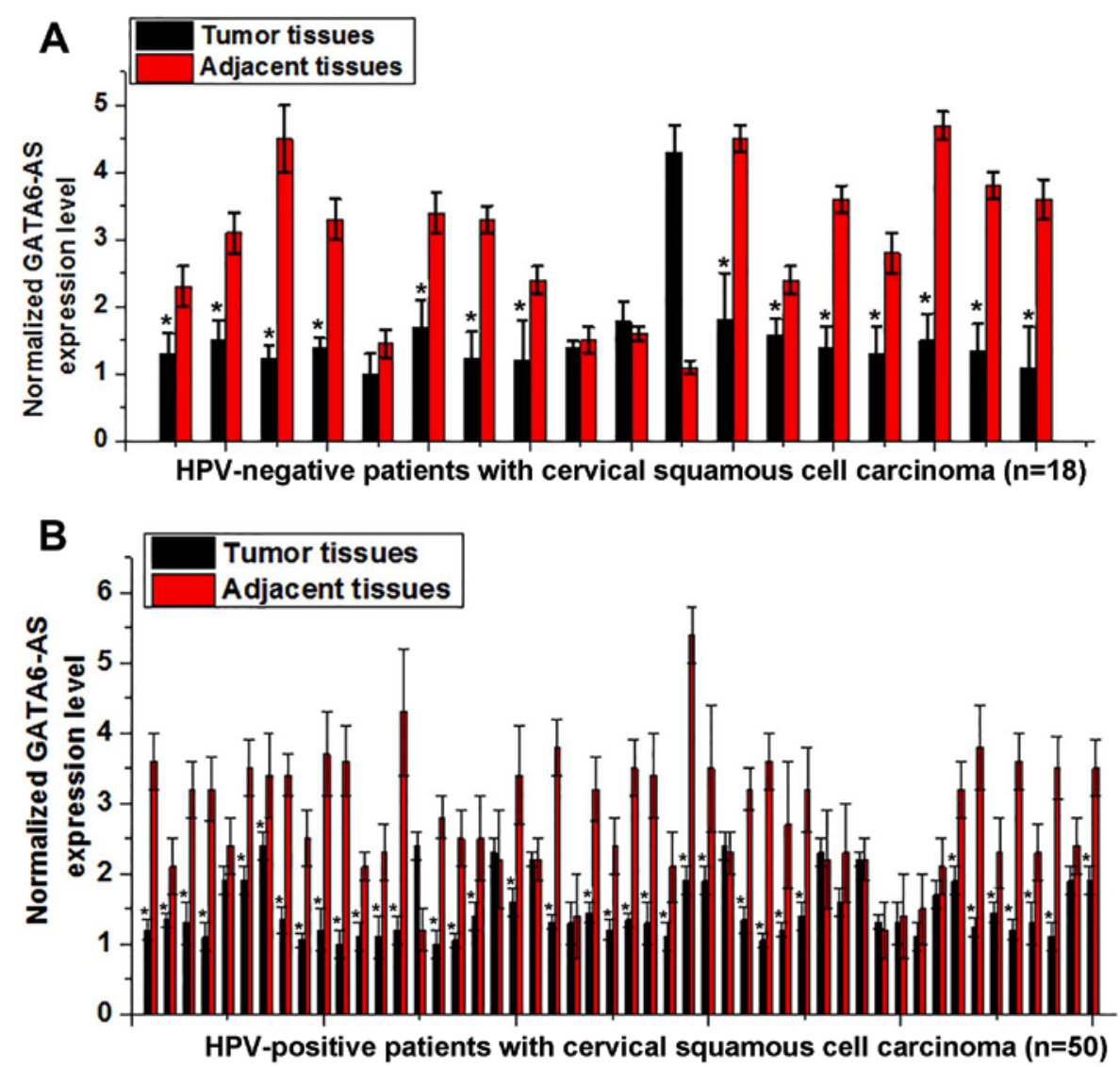

Figure 1. Expression of GATA6-AS in tumor tissues and adjacent normal tissues obtained from HPV-positive and HPV-negative patients. The normalized expression levels of GATA6-AS in tumor tissues and adjacent normal tissues is presented for (A) HPV-negative and (B) HPV-positive patients. "P<0.05 vs. adjacent tissues. HPV, human papillomavirus; GATA6-AS, GATA binding protein 6 antisense.
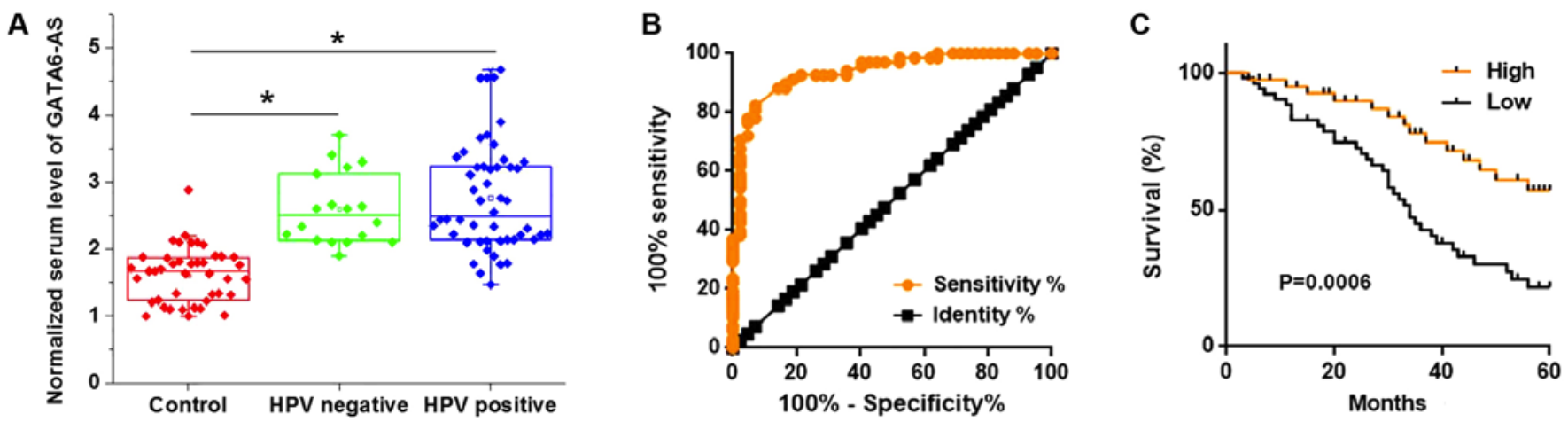

Figure 2. Serum levels of GATA6-AS in patients with cervical squamous cell carcinoma and healthy controls, and the diagnostic and prognostic value of GATA6-AS. (A) Comparison of serum GATA6-AS levels between patients with cervical squamous cell carcinoma and healthy controls. (B) Receiver operating characteristic curve analysis for the diagnostic value of serum GATA6-AS for cervical squamous cell carcinoma. (C) Survival analysis of patients with high or low serum levels of GATA6-AS. "P<0.05. HPV, human papillomavirus; GATA6-AS, GATA binding protein 6 antisense.

Effects of GATA6-AS-overexpression on MTK-1 expression in human cervical squamous cell carcinoma cells and normal cervical cell lines. MTK-1 is involved in the regulation of cell migration (12-14). The present study identified that GATA6-AS overexpression significantly downregulated the expression of MTK-1 in SiHa ( $\mathrm{P}<0.05$; Fig. 4A) and C33A ( $\mathrm{P}<0.05$; Fig. 4B) cells compared with the control and negative control groups. However, no significant differences in the expression of MTK-1 were observed in the normal cervical cell lines Ect1/E6E7 (Fig. 4C) and HCvEpC (Fig. 4D) following overexpression of
GATA6-AS, indicating that GATA6-AS may regulate MTK-1 only in cancer cells.

\section{Discussion}

Notably, the present study demonstrated that IncRNA GATA6-AS, which has an important regulatory role in the growth of endothelial cells (10), may also participates in the metastasis of cervical squamous cell carcinoma. It was determined that the role of GATA6-AS in cervical squamous cell 
Table I. Associations between serum levels of GATA6-AS and clinicopathological characteristics of patients with cervical squamous cell carcinoma.

\begin{tabular}{|c|c|c|c|c|c|}
\hline Characteristic & Cases, $\mathrm{n}$ & High GATA6-AS expression, $\mathrm{n}$ & Low GATA6-AS expression, $\mathrm{n}$ & $\chi^{2}$ & P-value \\
\hline \multicolumn{6}{|l|}{ Age, years } \\
\hline$>45$ & 38 & 17 & 21 & 0.95 & 0.33 \\
\hline$<45$ & 30 & 17 & 13 & & \\
\hline \multicolumn{6}{|c|}{ Primary tumor diameter, $\mathrm{cm}$} \\
\hline$>5$ & 16 & 7 & 9 & 1.64 & 0.44 \\
\hline$>3$ and $<5$ & 29 & 13 & 16 & & \\
\hline$>1$ and $\leq 3$ & 23 & 14 & 9 & & \\
\hline \multicolumn{6}{|c|}{ Tumor distant metastasis } \\
\hline Yes & 30 & 10 & 20 & 5.96 & $0.02^{\mathrm{a}}$ \\
\hline No & 38 & 24 & 14 & & \\
\hline \multicolumn{6}{|l|}{ Smoking } \\
\hline Yes & 16 & 7 & 9 & 0.33 & 0.57 \\
\hline No & 52 & 27 & 25 & & \\
\hline \multicolumn{6}{|l|}{ Drinking } \\
\hline Yes & 21 & 13 & 8 & 1.72 & 0.19 \\
\hline No & 47 & 21 & 26 & & \\
\hline
\end{tabular}

${ }^{\text {a }}<0.05$. GATA6-AS, GATA binding protein 6 antisense.

carcinoma is likely achieved by inhibiting cancer cell migration and invasion via inhibition of MTK-1 signaling.

It has been reported that IncRNAs serve key roles in normal physiological processes and pathological changes (15). Processes associated with the onset, development and progression of human cervical squamous cell carcinoma are accompanied with changes in expression patterns of certain IncRNAs (16). The IncRNA colon cancer associated transcript 2 (CCAT2) is significantly upregulated in cervical squamous cell carcinoma tissues compared with in healthy tissues, and the increased expression of CCAT2 is significantly associated with disease progression and poor prognosis (17). By contrast, maternally expressed 3 (MEG3), an oncogenic lncRNA, is downregulated in cervical squamous cell carcinoma, and upregulation of MEG3 inhibits cancer cell proliferation and promotes apoptosis (18). HPV infection is associated with altered expression levels of numerous IncRNAs (19). In the present study, the expression of lncRNA GATA6-AS was revealed to be significantly reduced in tumor tissues compared with adjacent healthy tissues in the majority of HPV-positive and HPV-negative patients. The current data suggest that IncRNA GATA6-AS may serve a role in cervical squamous cell carcinoma via an HPV-independent pathway.

The use of serum biomarkers has significantly improved the diagnosis of human diseases, including different types of cancer (20). In the present study, the serum levels of GATA6-AS were identified to be significantly higher in patients with cervical squamous cell carcinoma compared with healthy controls. However, no significant differences were revealed between HPV-negative and HPV-positive patients. Further analysis with a ROC curve revealed that serum GATA6-AS level can effectively distinguish patients with cervical squamous cell carcinoma from healthy controls. In addition, survival curve analysis demonstrated that a low serum level of GATA6-AS was significantly associated with poor survival. These data suggest that serum GATA6-AS level may be used for the diagnosis of cervical squamous cell carcinoma. It is understood that the expression of certain lncRNAs is affected by various factors, including aging (21), alcohol consumption (22) and tobacco abuse (23). In the present study, serum levels of GATA6-AS demonstrated no significant association with age, drinking habit or smoking habit, which indicates the high stability of serum GATA6-AS as a potential biomarker of disease. Notably, GATA6-AS is a newly identified lncRNA with an unknown expression pattern in other types of disease. Therefore, altered expression of GATA6-AS may indicate several possible diseases; multiple biomarkers may be combined to improve the diagnosis and prognosis.

The present study also demonstrated that serum levels of GATA6-AS were not significantly related to tumor size; however, a significant association with distant tumor metastasis was observed. In vitro cell migration and invasion assays revealed that GATA6-AS overexpression could inhibit the migration and invasion of cervical squamous cell carcinoma cells. The mitogen-activated protein kinase pathway serves critical roles in the development of malignancy (7). MTK-1, a key member of this pathway, participates in critical developmental processes and cellular functions (9). In addition, MTK-1 is involved in the regulation of cell migration (12-14). In the current study, GATA6-AS0 overexpression inhibited the expression of MTK-1 in cervical squamous cell carcinoma cells. Therefore, GATA6-AS may inhibit the progression of cervical squamous cell carcinoma by inactivating the mitogen-activated protein kinase pathway. However, the present study did not perform MTK-1-knockdown 


\section{A}

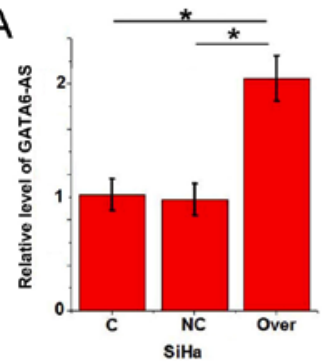

B
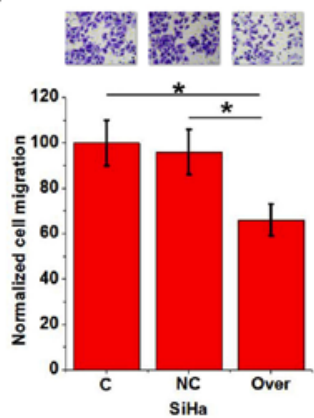

D
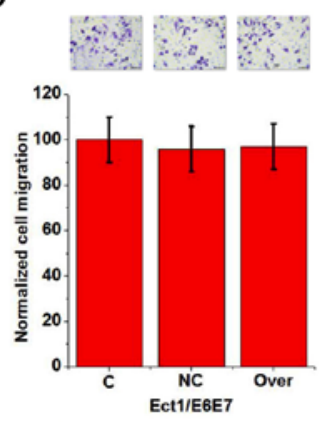
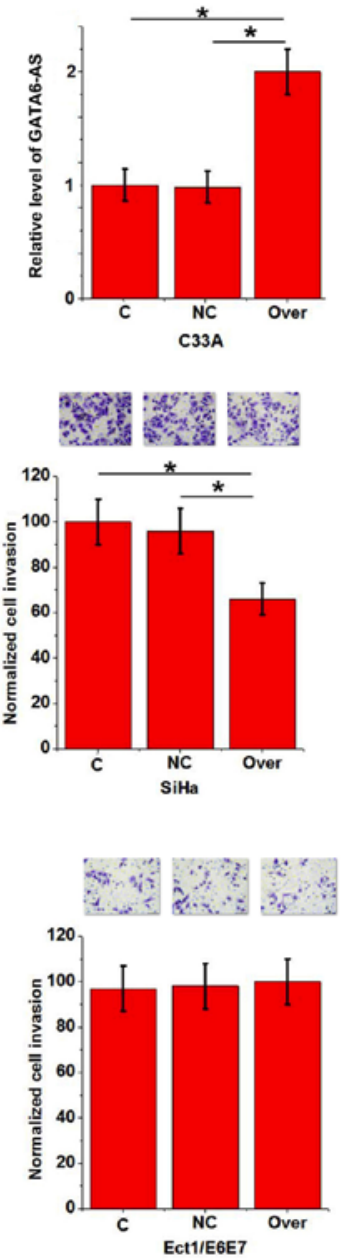

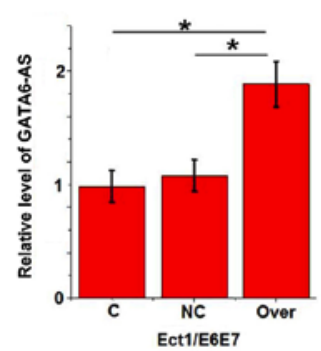

C
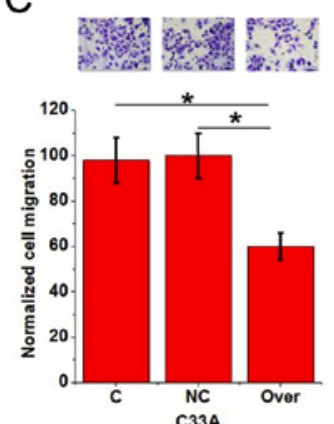

E
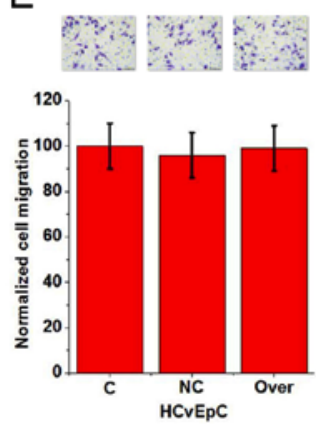
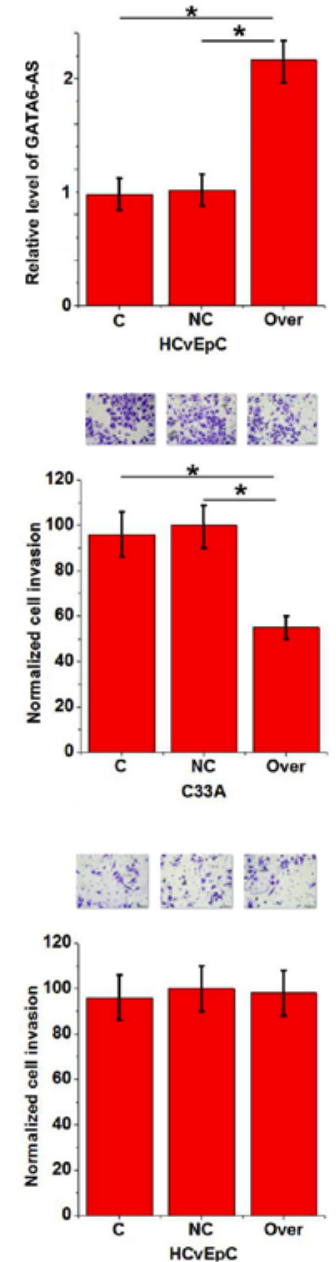

Figure 3. Effects of GATA6-AS overexpression on the migration and invasion of human cervical squamous cell carcinoma cell lines and normal cervical cell lines. (A) GATA6-AS expression levels were significantly higher in all cell lines following transfection. Exogenous expression of GATA6-AS inhibited the migration and invasion of the human cervical squamous cell carcinoma cell lines (B) SiHa and (C) C33A, but not the normal cervical cell lines (D) Ect1/E6E7 and (E) HCvEpC. " $\mathrm{P}<0.05$. GATA6-AS, GATA binding protein 6 antisense; C, control; NC, negative control; Over, GATA6-AS overexpression.
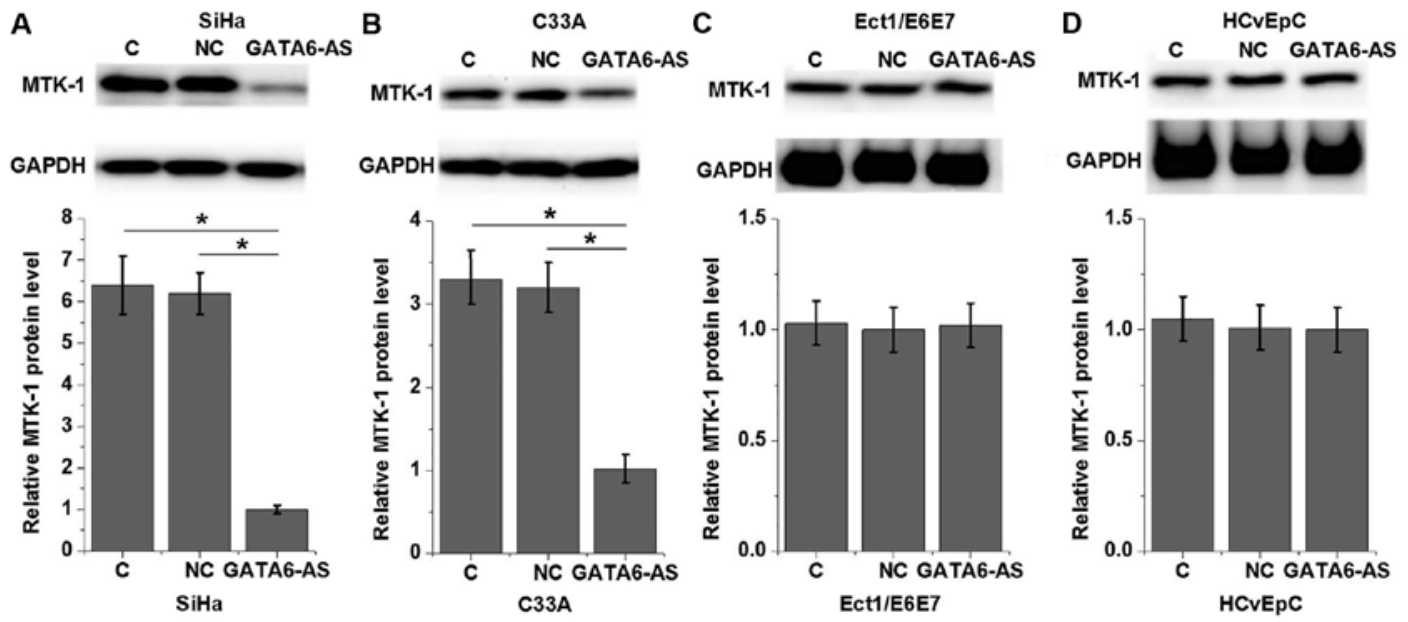

Figure 4. Effects of GATA6-AS-overexpression on MTK-1 expression in two human cervical squamous cell carcinoma cell lines and two normal cervical cell lines. GATA6-AS overexpression significantly downregulated the expression of MTK-1 in the two human cervical squamous cell carcinoma cell lines (A) SiHa and (B) C33A, but not in the two normal cervical cell lines (C) Ect1/E6E7 and (D) HCvEpC. " P<0.05. GATA6-AS, GATA binding protein 6 antisense; C, control; MTK-1, mitogen-activated protein kinase kinase 4; NC, negative control.

or MTK-1-overexpression experiments due to limited resources. Therefore, such investigations should be performed in the future.
Notably, GATA6-AS overexpression demonstrated no significant effects on the normal human cervical cell lines. 
Therefore, GATA6-AS may serve as a potential therapeutic target for cervical squamous cell carcinoma. However, the current study only included HPV-11, -16 and -18 strains, yet the expression of GATA6-AS in patients infected with other HPV strains remains unknown. Our future studies will attempt to include a larger sample size and investigate more HPV strains.

In conclusion, GATA6-AS expression was identified to be downregulated in cervical squamous cell carcinoma. Serum GATA6-AS levels may serve as a potential diagnostic and prognostic marker for cervical squamous cell carcinoma. In addition, GATA6-AS overexpression inhibited the migration and invasion of cancer cells, and downregulated MTK-1 expression. In summary, GATA6-AS may inhibit cancer cell migration and invasion by downregulating MTK-1.

\section{Acknowledgements}

Not applicable.

\section{Funding}

This study was supported by the National Natural Science Foundation of China (Beijing, China; grant no. 81470110).

\section{Availability of data and materials}

The datasets used and/or analyzed during the current study are available from the corresponding author on reasonable request.

\section{Authors' contributions}

LC and JZ designed the study. LC, XW, LS and DY performed the experiments. QT assisted the experiments and collected the data. JZ drafted the manuscript. All authors have read and approved this manuscript.

\section{Ethics approval and consent to participate}

The present study was approved by the Ethics Committee of Union Hospital, Tongji Medical College, Huazhong University of Science and Technology (Wuhan, China). Written informed consent was obtained from all participants.

\section{Patient consent for publication}

Not applicable.

\section{Competing interests}

The authors declare that they have no competing interests.

\section{References}

1. Ferlay J, Soerjomataram I, Dikshit R, Eser S, Mathers C, Rebelo M, Parkin DM, Forman D and Bray F: Cancer incidence and mortality worldwide: Sources, methods and major patterns in GLOBOCAN 2012. Int J Cancer 136: E359-E386, 2015.

2. Yang PM, Chou CJ, Tseng SH and Hung CF: Bioinformatics and in vitro experimental analyses identify the selective therapeutic potential of interferon gamma and apigenin against cervical squamous cell carcinoma and adenocarcinoma. Oncotarget 8 : 46145-46162, 2017.
3. Crosbie EJ, Einstein MH, Franceschi S and Kitchener HC: Human papillomavirus and cervical cancer. Lancet 382 : 889-899, 2013.

4. Burd EM: Human papillomavirus and cervical cancer. Clin Microbiol Rev 16: 1-17, 2003

5. Hildesheim A, Gonzalez P, Kreimer AR, Wacholder S, Schussler J, Rodriguez AC, Porras C, Schiffman M, Sidawy M, Schiller JT, et al: Impact of human papillomavirus (HPV) 16 and 18 vaccination on prevalent infections and rates of cervical lesions after excisional treatment. Am J Obstet Gynecol 215: 212. e1-212.e15, 2016.

6. Rodríguez-Carunchio L, Soveral I, Steenbergen RD, Torné A, Martinez S, Fusté P, Pahisa J, Marimon L, Ordi J and del Pino M: HPV-negative carcinoma of the uterine cervix: A distinct type of cervical cancer with poor prognosis. BJOG 122: 119-127, 2015.

7. Yang $M$ and Huang CZ: Mitogen-activated protein kinase signaling pathway and invasion and metastasis of gastric cancer. World J Gastroenterol 21: 11673-11679, 2015.

8. Peng S,Geng J,Sun R,TianZand WeiH:Polyinosinic-polycytidylic acid liposome induces human hepatoma cells apoptosis which correlates to the up-regulation of RIG-I like receptors. Cancer Sci 100: 529-536, 2009.

9. Fredholm S, Litvinov IV, Mongan NP, Schiele S, Willerslev-Olsen A, Petersen DL, Krejsgaard T, Sibbesen N, Nastasi C, Bonefeld CM, et al: The expression of IL-21 is promoted by MEKK4 in malignant t cells and associated with increased progression risk in cutaneous T-cell lymphoma. J Invest Dermatol 136: 866-869, 2016.

10. Tasharrofi B and Ghafouri-Fard S: Long non-coding RNAs as regulators of the mitogen-activated protein kinase (MAPK) pathway in cancer. Klin Onkol 31: 95-102, 2018.

11. Neumann P, Jaé N, Knau A, Glaser SF, Fouani Y, Rossbach O, Krüger M, John D, Bindereif A, Grote P, et al: The lncRNA GATA6-AS epigenetically regulates endothelial gene expression via interaction with LOXL2. Nat Commun 9: 237, 2018.

12. Sollome JJ, Thavathiru E, Camenisch TD and Vaillancourt RR: HER2/HER3 regulates extracellular acidification and cell migration through MTK1 (MEKK4). Cell Signal 26: 70-82, 2014.

13. Allison P, Espiritu D and Camenisch TD: BMP2 rescues deficient cell migration in Tgfbr3(-/-) epicardial cells and requires Src kinase. Cell Adh Migr 10: 259-268, 2016.

14. Du J, Zhou S, Wang L, Yu M and Mei L: Downregulation of ERBB3 decreases the proliferation, migration and invasion of cervical cancer cells though the interaction with MTK-1. Oncol Lett 16: 3453-3458, 2018.

15. Fatica A and Bozzoni I: Long non-coding RNAs: New players in cell differentiation and development. Nat Rev Genet 15: 7-21, 2014.

16. Sharma $S$ and Munger K: Expression of the cervical carcinoma expressed PCNA regulatory (CCEPR) long noncoding RNA is driven by the human papillomavirus E6 protein and modulates cell proliferation independent of PCNA. Virology 518: 8-13, 2018.

17. Chen X, Liu L and Zhu W: Up-regulation of long non-coding RNA CCAT2 correlates with tumor metastasis and poor prognosis in cervical squamous cell cancer patients. Int J Clin Exp Pathol 8: 13261-13266, 2015.

18. Zhang J, Yao T, Wang Y, Yu J, Liu Y and Lin Z: Long noncoding RNA MEG3 is downregulated in cervical cancer and affects cell proliferation and apoptosis by regulating miR-21. Cancer Biol Ther 17: 104-113, 2016.

19. Nohata N, Abba MC and Gutkind JS: Unraveling the oral cancer lncRNAome: Identification of novel lncRNAs associated with malignant progression and HPV infection. Oral Oncol 59: 58-66, 2016.

20. Lutz AM, Willmann JK, Cochran FV, Ray P and Gambhir SS: Cancer screening: A mathematical model relating secreted blood biomarker levels to tumor sizes. PLoS Med 5: e170, 2008.

21. Grammatikakis I, Panda AC, Abdelmohsen K and Gorospe M: Long noncoding RNAs (lncRNAs) and the molecular hallmarks of aging. Aging (Albany NY) 6: 992-1009, 2014.

22. Mayfield RD: Emerging roles for ncRNAs in alcohol use disorders. Alcohol 60: 31-39, 2017.

23. Wang J, Qiu M, Xu Y, Li M, Dong G, Mao Q, Yin R and Xu L: Long noncoding RNA CCAT2 correlates with smoking in esophageal squamous cell carcinoma. Tumour Biol 36: 5523-5528, 2015. 